\title{
O ABORTO COMO CAUSA DE MORTALIDADE MATERNA: UM PENSAR PARA O CUIDADO DE ENFERMAGEM
}

\author{
Abortion as a cause of maternal death: a thought for nursing care \\ El aborto como causa de mortalidad materna: una reflexión \\ sobre el cuidado de enfermería
}

\section{RESUMO}

0 aborto é um tema polêmico e um sério problema de saúde pública mundial, responsável pela manutenção das altas taxas de mortalidade materna em muitos países em desenvolvimento. No Brasil, por se tratar de um ato ilegal, muitas mulheres que não desejam manter uma gestação acabam por procurar clínicas clandestinas, submetendo-se ao aborto em condições precárias, 0 que acarreta graves consequências à sua saúde física e psicológica e à própria vida. Como enfermeiras, reconhecemos a magnitude das questões que permeiam as discussões sobre o abortamento e a mortalidade materna no cenário das políticas de saúde que envolvem a área da saúde da mulher e, dessa forma, nos propomos, com este artigo, realizar uma reflexão acerca do abor to como causa de mortalidade materna.

Palavras-chave: Enfermagem. Aborto. Mortalidade Materna. Saúde da Mulher.

\begin{abstract}
Abortion is a polemic theme and a serious public health problem in the world, responsible for the maintenance of high maternal death rates in many developing countries. In Brazil, even though it is an illegal act, many women who do not wish to maintain their pregnancy end up seeking underground clinics where they undergo abortions in precarious conditions, carrying with it grave consequences to their physical and mental health and even their own lives. As nurses, we recognize the magnitude of these questions that permeate discussions about abortion and maternal death regarding health policy involving the area of women's health, and for this reason intend to, with this article, induce a reflection concerning abortion as a cause of maternal death.
\end{abstract}

Key Words: Nursing. Abortion. Maternal death. Women's health

\section{Resumen}

El aborto es un tema polémico y un serio problema de salud pública mundial, responsable por el mantenimiento de las altas tasas de mortalidad materna en muchos países en desarrollo. En Brasil, por tratarse de un delito, muchas mujeres que no desean continuar con un embarazo acaban buscando clínicas clandestinas sometiéndose al aborto en condiciones precarias, lo que ocasiona graves consecuencias a su salud física y psicológica y a su propia vida. Como enfermeras, reconocemos la magnitud de las cuestiones que engloban las discursiones sobre el aborto y la mortalidad materna en el escenario de las políticas de salud relacionadas con el área de la salud de la mujer y, de esta forma, nos proponemos, con este artículo, realizar una reflexión sobre el aborto como causa de mortalidad materna.

Palabras clase: Enfermería. Aborto. Mortalidad materna. Salud de la mujer.

${ }^{1}$ Enfermeira, Mestre em Enfermagem, Aluna do Curso de Doutorado da Escola de Enfermagem da Universidade de São Paulo. Brasil. E-mail: selisvane@usp.br, Enfermeira, Doutora em Enfermagem. Professora Titular do Departamento de Enfermagem Materno-Infantil e Psiquiátrica da Escola de Enfermagem da Universidade de São Paulo. Brasil. E-mail: merighi@usp.br 
O aborto como causa de mortalidade materna

Domingos SRF, Merighi MAB

\section{INTRODUČ̃̃}

Em pleno século XXI, o aborto ainda constitui um problema de saúde pública e é um dos temas de maior destaque nas discussões que envolvem a área da saúde da mulher e, especificamente, a mortalidade materna. Trata-se de um assunto polêmico que articula diversas posições e conflitos pessoais, culturais, religiosos e sociais.

Definido pela Organização Mundial de Saúde (OMS) como a expulsão do concepto com peso inferior a $500 \mathrm{~g}$, com cerca de 20-22 semanas completas de gestação, ocorre de forma precoce, antes da $13^{\mathrm{a}}$ semana, e tardia, entre a $13^{\mathrm{a}}$ e a $22^{\mathrm{a}}$ semana de gestação. 'É classificado por sua etiologia como espontâneo ou provocado.

0 aborto provocado representa um sério problema de saúde mundial, pois é responsável pela maioria dos óbitos relacionados à prática do abortamento. ${ }^{2}$

Na última década, o abor to foi amplamente debatido na Conferência Internacional de População e Desenvolvimento (CIPD) em 1994, no Cairo, e na Conferência Mundial sobre a mulher realizada em 1995, em Beijing. 0 aborto realizado em condições inseguras foi incluído no Plano de Ação da Conferência do Cairo (parágrafo 8.25) como questão de saúde pública, e os governos signatários, entre eles o Brasil, assumiram 0 compromisso de implementar serviços para reduzir a morbidade e a mortalidade por aborto em seus países. ${ }^{3}$ No entanto, nos dias atuais, no Brasil e na maior parte da América Latina, 0 aborto clandestino e inseguro é ainda um grave problema de saúde pública, e sua solução é um desafio que perpassa a exigência de medidas urgentes no processo de descriminalização. ${ }^{4}$

De acordo com o Sistema Único de Saúde (SUS), a incidência de óbitos por complicações do aborto oscila em torno de $12,5 \%$, ocupando o terceiro lugar entre as causas de mortalidade materna com variações entre os estados

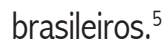

Segundo dados da OMS, o nosso país lidera as estatísticas de abortamento provocado em todo o mundo com um total de quatro milhões por ano. Em um contingente de 36 milhões de mulheres, uma em cada nove mulheres brasileiras recorre ao abor to como meio para terminar uma gestação que não foi planejada ou indesejada. ${ }^{6}$ No entanto, vale a pena ressaltar que não se sabe, exatamente, quantas mulheres vivenciam o aborto ou quantas morrem anualmente por causas relacionadas às suas complicações, pois, por ser ilegal em muitos países do mundo, inclusive no Brasil, ocorre predominantemente na clandestinidade e em condições precárias.

A criminalização do aborto coloca as mulheres, na maioria das vezes, nas mãos de pessoas despreparadas, para realização de um aborto inseguro, e em condições clandestinas, pondo em risco sua própria vida. Além do mais, a clandestinidade transforma o aborto em um negócio lucrativo, garantindo a impunidade para aqueles que o realizam. Quem tem maior
Esc Anna Nery Rev Enferm 2010 jan-mar; 14 (1): 177-81

poder aquisitivo utiliza as clínicas especializadas e têm acesso a uma assistência qualificada, enquanto quem não tem condições financeiras busca pessoas não habilitadas e métodos abortivos rudimentares, que podem levar a graves complicações e à morte. $^{2}$

As complicações do aborto são importantes causas de morbidade e mortalidade das mulheres, principalmente nos países em desenvolvimento. No entanto, sabe-se que a incidência da morbidade relacionada com o aborto provocado em condições precárias é alta e que tais dados são mais difíceis de se obter do que aqueles relativos à mortalidade. ${ }^{7}$

Como enfermeiras atuantes na área da saúde da mulher, tanto na docência como na assistência, convivemos com mulheres em situações de abortamento que buscam nos serviços de saúde atendimento para suas necessidades, na maioria das vezes em condições clínicas graves, tais como sangramento vaginal abundante, fortes dores, febre e outros que caracterizam complicações por abortamento e que colocam em risco suas vidas. Não bastassem tais problemas clínicos, muitas delas, em seu modo peculiar de ser, demonstram sofrimento relacionado à perda e culpa pela interrupção da gestação, e, quando não encontram nos serviços de saúde profissionais capazes de ouvilas em suas queixas e de prestar cuidado sem julgamentos, esse sofrimento parece se intensificar.

Por reconhecermos a magnitude das questões que permeiam as discussões sobre o abortamento e a mortalidade materna no cenário das políticas de saúde que envolvem a área da saúde da mulher, nos propomos, com este artigo, realizar uma reflexão acerca do aborto como causa de mortalidade materna. Acreditamos que os modos de pensar sobre o aborto disseminados pela sociedade são pouco discutidos e, consequentemente, repassados de geração para geração conforme representam para cada um, tornando-os, assim, de difícil investigação.

\section{O aborto e a mortalidade materna no contexto da saúde mundial e brasileira}

As legislações vigentes em todos os países do mundo têm, umas mais e outras menos, acompanhado as constantes mudanças e evoluções sociais e científicas. Existem países que não permitem o aborto, exceto quando há risco para a vida da mãe; países que permitem o aborto, mas com restrições; e países que permitem o aborto, independentemente do motivo que o condiciona. ${ }^{8}$

Com relação ao aborto inseguro, destaca-se que a grande maioria acontece em países onde as leis do aborto são muito restritivas e onde ele é considerado ilegal. Dessa forma, muitas mulheres, diante de uma gravidez não planejada e indesejada, recorrem a meios clandestinos de realizar o aborto e acabam colocando em risco a própria vida.

No Brasil, o Código Penal Brasileiro, promulgado em 1940, prevê a prática legal do abor to apenas quando não há outro meio de salvar a vida da gestante ou quando a gravidez resultar 
de estupro ou incesto. Há, também, muito raramente, casos de má formação congênita em que a interrupção da gravidez é autorizada pela justiça. ${ }^{9}$

No entanto, a ilegalidade do aborto no Brasil não tem impedido sua prática. De acordo com a OMS, no Brasil, $31 \%$ dos casos de gravidez terminam em abortamento. De acordo com as estimativas, anualmente ocorrem 1,4 milhão de abortamento espontâneo e inseguro, com uma taxa de 3,7 abortos para cada 100 mulheres em idades de 15 a 49 anos. Como reflexo dessa situação, em 2004, 243.998 internações na rede SUS foram por curetagens pós-abortamento, correspondentes aos casos de complicações. As curetagens são o segundo procedimento obstétrico mais realizado nas unidades de internação, sendo superados apenas pelos partos normais. Além do mais, o abortamento é uma importante causa de mor talidade materna no país. Em 2001, aconteceram 9,4 mortes de mulheres por abortamento por 100 nascidos vivos. ${ }^{10}$

A mortalidade materna, ou seja, "a morte de uma mulher que ocorre durante a gestação ou dentro de um período de 42 dias após o término da gestação, independente da duração ou localização da gravidez", 7 de acordo como o Ministério da Saúde, é uma das mais graves violações aos direitos das mulheres, por se tratar de algo evitável em aproximadamente $92 \%$ dos casos e ocorrer predominantemente nos países em desenvolvimento. ${ }^{11}$

Ao fazer uma breve retrospectiva da situação da mortalidade materna no mundo e no Brasil, a OMS coloca que, em 1990, 585.000 mulheres em todo o mundo morreram por complicações relacionadas ao ciclo gravídico-puerperal, sendo que apenas $5 \%$ delas viviam em países desenvolvidos. 0 Canadá e os Estados Unidos apresentaram índices inferiores a 9 óbitos maternos por cada 100.000 nascidos vivos. Já em países como Bolívia, Peru e Haiti, esses dados eram de mais de 200 óbitos. Em toda a América Latina, cerca de 28.000 mulheres morrem por ano devido a complicações da gravidez, parto e puerpério, sendo a grande maioria desses óbitos evitáveis. No Brasil, a subinformação e o sub-registro das declarações das causas de óbitos dificultam o real monitoramento da mortalidade materna. Levando-se em conta os óbitos declarados, durante a década de 80 , houve um declínio na taxas de mortalidade materna, permanecendo inalterada no período de 1988 a 1997. De 1999 a 2001 houve uma nova queda nesses valores e, em 2003, a razão de mortalidade materna foi de 51,7 óbitos por 100.000 nascidos vivos. Os maiores valores são encontrados nas regiões nordeste e centro-oeste; e os menores, na região sudeste. ${ }^{11}$

Em estudo realizado em Teresina/PI sobre o perfil da mortalidade materna em maternidade pública, as autoras ressaltam que, embora o Ministério da Saúde tenha investido em várias áreas de assistência à saúde da mulher, poucas mudanças têm ocorrido no contexto social da saúde feminina. Como resultado desse processo, observamos os altos coeficientes de mortalidade materna e a ineficiência na qualidade dos serviços de saúde na área de atenção à saúde da mulher. No entanto, destacamos que a morte materna deve ser encarada como fato possível de intervenções dos profissionais de saúde que lidam com mulheres, pois não é mais admissível que em pleno século XXI a contabilização de tantos óbitos relacionados à reprodução humana. ${ }^{12}$

Nesse contexto, ressalta-se a importância dos comitês de mortalidade materna; especificamente no Brasil, a implantação dos comitês estaduais de morte materna tem sido adotada como estratégia do Programa de Assistência Integral à Saúde da Mulher (PAISM), delineada em 1984 pelo Ministério da Saúde. No entanto, foi a partir de 1987 que o desenvolvimento dos comitês de fato aconteceu. No período de 1993 a 1996, em todos os estados brasileiros foram implantados os comitês de morte materna, e, em 1994, foi instituída a Comissão Nacional de Morte Materna. Em 2005, os 27 comitês já estavam implantados. ${ }^{11}$

No entanto, apesar desses avanços, o Brasil continua sendo um dos países do mundo em subdesenvolvimento que possui as mais altas taxas de mortalidade materna, ocupando a $65^{\mathrm{a}}$ posição no ranking mundial de mortalidade materna. ${ }^{4}$

\section{O aborto como causa de mortalidade materna}

0 aborto, conforme exposto anteriormente, é considerado importante causa de mortalidade materna. Entretanto, sabemos que, com relação ao abortamento, o grande problema nas discussões das questões que envolvem a mortalidade materna está relacionado, principalmente, ao abortamento realizado em condições inseguras.

No mundo, aproximadamente $15 \%$ dos casos de mortalidade materna são decorrentes de abortos inseguros, chegando a 50\% em algumas áreas, o que poderia ser prevenido por meio da ampliação do acesso das mulheres ao tratamento das complicações decorrentes do aborto, assim como por procedimentos abortivos seguros e serviços com foco no planejamento familiar. ${ }^{7}$ Destaca-se, ainda, que a maioria dos estudos com mulheres em situação de abortamento é realizada em hospitais e que a grande maioria dos abortos provocados ocorre em comunidades de baixa renda.

Em estudo realizado pelo Ministério da Saúde sobre mortalidade em mulheres com ênfase na mortalidade materna, o abortamento foi responsável por $11,4 \%$ do total das mortes maternas e por $17 \%$ das causas obstétricas diretas nas capitais brasileiras, sendo que uma parcela significativa desses casos correspondeu ao aborto provocado. ${ }^{13}$

No que diz respeito às complicações, é importante ressaltar que as complicações físicas do aborto podem estar presentes em decorrência do próprio processo de abortamento ou pelos procedimentos realizados para tratamentos. Essas complicações podem ser classificadas em grandes hemorragias, perfurações uterinas decorrentes de sondas ou cânulas, ulcerações do colo ou vagina por uso de comprimidos, infecções, esterilidade secundária a salpingite, salpingite crônica, algias pélvicas, 
O aborto como causa de mortalidade materna

transtornos menstruais e complicações obstétricas, tais como inserção anormal da placenta, abortamentos habituais, partos prematuros, dentre outras. $^{14}$

Com relação ao tratamento para as formas clínicas do aborto, o Ministério da Saúde estabelece classificação e conduta conforme o tipo de abortamento e de acordo com os sintomas apresentados pelas mulheres, tais como uso de medicamentos, aspiração manual intrauterina nos casos de abortamento até 12 semanas, dilatação do canal cervical, curetagem uterina, dentre outros. ${ }^{3}$

Em estudo realizado sobre mortalidade materna em Recife, nos anos de 1992 e 1993, foi possível constatar que as complicações do aborto representaram quase $12 \%$ das mortes, sendo todos esses óbitos decorrentes da interrupção voluntária da gestação. Para os autores, esse dado mostra uma falha nos serviços de saúde com relação ao planejamento familiar. 0 estudo ressalta que a proibição da venda e a dificuldade da obtenção para promover a interrupção de uma gravidez provavelmente estão relacionadas ao reaparecimento de casos de abortamento complicado. ${ }^{15}$

Em um outro estudo sobre as mortes por aborto no município de São Paulo ocorridas nos anos de 1997 e 1998, foi possível identificar a ocorrência de 21 óbitos de mulheres por aborto em um conjunto de 260 óbitos maternos. Os dados mostram que essas mulheres eram jovens, solteiras, residentes na periferia de São Paulo, a maioria trabalhadora e com baixa escolaridade. A autora menciona que a trajetória que culminou com o óbito foi marcada pela carência de informações e por atendimentos em serviços de saúde não resolutivos. ${ }^{6}$

Os resultados de uma pesquisa recente sobre a epidemiologia do aborto inseguro realizada em uma comunidade favelar em São Paulo mostraram que, em uma população de 375 mulheres, houve 144 abortos, com uma média de 1,55 aborto por mulher. 0 aborto provocado (82 casos) e o espontâneo (62 casos) mostraram-se inseguros, pois em mais de $93 \%$ dos casos os abortos foram sofridos ou provocados nas casas das próprias mulheres, em locais sem condições de higiene, sem assepsia e sem nenhum tipo de acompanhamento, e, quando provocados, o foram pelas próprias mulheres ou por pessoas sem a mínima capacitação. Um total de $94,12 \%$ das mulheres que provocaram o abor to e $83,3 \%$ das que sofreram um aborto espontâneo referiram complicações pós-aborto e $82,7 \%$ do total delas mencionaram internação referente ao abortamento. Para as autoras, as complicações decorrentes do pós-aborto ou da morbidade relacionada ao aborto refletem na saúde propriamente dita da mulher, na possibilidade de atendimento pelos serviços de saúde, na sobrecarga hospitalar e nos custos das internações. ${ }^{4}$

A prevenção da morbimortalidade materna relacionada ao abor to depende da existência de serviços de saúde estruturados nos diversos níveis de assistência para garantir atendimento às mulheres.
Esc Anna Nery Rev Enferm 2010 jan-mar; 14 (1): 177-81

Na tentativa de buscar caminhos e soluções para reduzir o problema da mor talidade materna, algumas estratégias têm merecido destaque: adequado sistema de registro de nascimentos e mortes, implantação de comitês de mortalidade materna, incentivo ao planejamento familiar, qualidade na atenção pré-natal, capacitação profissional, incremento tecnológico, treinamento de parteiras tradicionais e institucionalização do parto. Nesse contexto, o planejamento familiar, quando oferecido de forma contínua e prolongada, pode contribuir para a redução do número de gestações indesejadas e abortos ilegais e contribuir para a redução da mortalidade materna. ${ }^{16}$

Além do mais, no Brasil emerge uma necessidade cada vez maior de estudos sobre o abortamento inseguro, clandestino, principalmente em populações de baixa renda, nas quais há um número maior de casos de aborto nas taxas de morbimortalidade materna e onde se faz mais necessário trabalhar o planejamento familiar preventivo. ${ }^{4}$ É preciso considerar que o aborto provocado é uma realidade que atinge, com frequência alarmante, a mulher brasileira e que, portanto, merece um tratamento mais sério do que aquele que tem recebido até agora em nossa sociedade.

\section{CONSIDERAÇÕES FINAIS}

Ao realizar um aborto, a mulher guarda sua dor em silêncio ou, no máximo, compartilha com pessoas de sua intimidade; em geral, ela não encontra apoio nos serviços de saúde. Dar oportunidade para que a mulher fale sobre suas emoções não só ajuda, mas fornece subsídios para o planejamento dos cuidados necessários e específicos. A perda mal resolvida pode se manifestar de diferentes formas nos comportamentos futuros, ${ }^{3}$ além de contribuir para a manutenção do problema ou, quem sabe, agravá-lo.

0 tratamento humanizado pelos profissionais de saúde é imprescindível, devendo ser demonstrado por meio do respeito à opção pelo aborto, considerando o período de internação como oportunidade de escuta sobre os fatores psicossociais que envolvem essa decisão e para a promoção do conhecimento com base na troca de saberes e não de oposição. ${ }^{2}$

As reflexões feitas neste estudo propiciaram oportunidade ímpar para fortalecer em nós, enfermeiros, a crença na necessidade de ampliarmos o olhar para o sujeito das nossas ações. Vale ressaltar que, durante nossa formação, aprendemos que as situações da vida da pessoa lhe conferem um modo especial de vivenciar cada experiência. Os componentes socioculturais e econômicos podem determinar modos diferentes de cursar cada fase da vida. Por esse motivo acreditamos que devemos considerar esses fatores, bem como a subjetividade e as experiências de cada mulher, buscando, a partir daí, apreender as suas necessidades.

É imprescindível refletirmos sobre os significados atribuídos pelas mulheres que vivenciam a situação de abortamento e interpretarmos os sinais e sintomas não apenas como algo 
orgânico. Essa atitude do profissional certamente pode tornar o cuidado mais abrangente, menos mecanicista e reducionista, resgatando a unidade humana por meio de uma prática que aproxima o saber da sensibilidade, propiciando, assim, o reencontro da ciência com o humano.

A qualidade da atenção implica um espaço integrado e sinérgico de todos os níveis gestores para a oferta de serviços que garantem: acolhimento, informação, aconselhamento, competência profissional, tecnologia apropriada disponível e relacionamento pessoal pontuado no respeito à dignidade $\mathrm{e}$ aos direitos sexuais e reprodutivos. Com frequência, no pósabortamento, as mulheres precisam de uma equipe que compreenda suas necessidades, não somente físicas, mas também sociais e psicológicas. ${ }^{3,5}$

Atualmente as questões relacionadas à saúde da mulher continuam sendo tema de interesse nas discussões que envolvem grupos populacionais, pois muitas das condições de morbidade e mortalidade feminina ainda constituem os principais problemas

\section{REFERÊNCIAS}

1- Ministério da Saúde (BR). Secretaria de Atenção a Saúde. Departamento de Ações Programáticas e Estratégicas. Urgências e emergências maternas. Guia para diagnóstico e conduta em situaç̃̃es de risco de morte materna. Brasília (DF); 2000.

2- Souza VLC, Corrêa MSM, Souza SL, Beserra MA. 0 aborto entre adolescentes. Rev Latino-am Enfermagem 2001 mar; 9(2): 42-7.

3- Ministério da Saúde (BR). Secretaria de Políticas de Saúde. Área Técnica de Saúde da Mulher. Parto, aborto e puerpério: assistência humanizada à mulher. Brasília (DF); 2001.

4- Fusco CLB, Andreoni S, Silva RS. Epidemiologia do aborto inseguro em uma população em situação de pobreza - Favela Inajar de Souza, São Paulo. Rev Bras Epidemiol [on-line] 2008 mar. [citado 26 maio 2008]; 11 (1): [aprox.12 telas]. Disponível em: http://www.scielo.br.

5- Mariutti MG, Furegato ARF, Scatena MCM, Silva, L. Relação de ajuda entre o enfermeiro e mulheres em abortamento espontâneo. Cienc Cuid Saude, Maringá 2005 jan/abr; 4(1): 83-8.

6- Bittencourt MLM. As mortes por aborto no município de São Paulo: estudo epidemiológico sob o enfoque de gênero. [dissertação de mestrado]. São Paulo (SP): Escola de Enfermagem/ USP; 2000.

7- Organização Mundial de Saúde-OMS. Complicaciones del aborto: directrices técnicas y gestoriales de prevención y tratamiento. Ginebra; 1995.

8- Duarte GA, Alvarenga AT, Osis MJMD, Faúndes A, Hardy E. Perspectiva masculina acerca do aborto provocado. Rev Saude Publica. [on-line] 2002 jun; [citado 23 out 2007]; 36(3). [aprox. 12 telas]. Disponível em http://www.scielo.br.

9- César JA, Gomes G, Horta BL, Oliveira AK, Saraiva AKO, Pardo DO, et al. Opinião de mulheres sobre a legalização do aborto em município de porte médio no sul do Brasil. Rev Saude Publica. [on-line]; 1997 dez [citado citado 23 out 2007]; 31(6). [aprox.12 telas]. Disponível em: http://www.scielo.br. de saúde pública no mundo e principalmente no Brasil. Cabe destacar que a atenção à saúde da mulher envolve a compreensão de aspectos biológicos, epidemiológicos, sociais, culturais, éticos e antropológicos articulados em um contexto de atuação de uma equipe multiprofissional.

Vale acrescentar que, apesar dos grandes avanços, ainda é necessário discutir formas e caminhos para solucionar problemas acerca da morbidade feminina. A mortalidade materna é considerada um indicador de qualidade da atenção à saúde, e seus elevados índices em países subdesenvolvidos como o Brasil retratam a necessidade urgente de melhorias nessa área.

A redução da mortalidade materna requer, além das questões aqui levantadas, uma emergente vontade política e educacional para investir nos serviços de saúde que prestam atendimento às mulheres. Caso contrário, pouco se poderá fazer para mudar os atuais índices de mortalidade materna brasileiros e a assistência à saúde da mulher no contexto do abortamento.

10- Ministério da Saúde (BR). Ministério da Saúde lança política nacional que amplia acesso ao planejamento familiar - 22/03/2005. [citado 26 maio 2008]. Disponível em: http://portal.saude.gov.br/ portal/aplicacoes/noticias/noticias

11- Ministério da Saúde (BR). Secretaria de Atenção a Saúde. Departamento de Ações Programáticas e Estratégicas. Manual dos comitês de mortalidade materna. $3^{\mathrm{a}}$ ed. Brasília (DF); 2007.

12- Nascimento FM, Bezerra RLA, Dantas MFS, Nery IS. Perfil da mortalidade materna em maternidade pública de Teresina-PI no período de 1996 a 2000: uma contribuição da enfermagem. Esc Anna Nery Rev Enferm 2007 set; 11(3): 472-78.

13- Ministério da Saúde (BR). Secretaria de Atenção a Saúde. Departamento de Ações Programáticas e Estratégicas. Estudo da mortalidade de mulheres de 10 a 49 anos, com ênfase na mortalidade materna: relatório final. Brasília (DF); 2006.

14- Carvalho GM. Enfermagem em obstetrícia. São Paulo (SP): EPU; 1990.

15- Cecatti JG, Albuquerque RM, Hardy E, Faúndes A. Mortalidade materna em Recife: causas de óbitos maternos. Rev Bras Ginecol Obstetr [on-line]. 1998 jan/fev; [citado 16 maio 2008] 20(1). [aprox.12 telas]. Disponível em: http://www.scielo.br.

16- Alencar Júnior CA. Os elevados índices de mortalidade materna no Brasil: razões para sua permanência. Rev Bras Ginecol Obstetr [on-line]. 2006 jul; [citado 18 mar 2008]. 28(7). [aprox.12 telas]. Disponivel em: http://www.scielo.br. 